Artículo de investigación

\title{
Efectos de la generación de energía hidroeléctrica en la reproducción de Prochilodus magdalenae (Prochilodontidae): evidencia de la respuesta endocrina
}

\author{
Daniela De Fex-Wolf1,2* $₫(\mathbb{0}$ M.Sc; Silvia López-Casas $1,3 \otimes(\mathbb{0}$ Ph.D; \\ Luz Fernanda Jiménez-Segura ${ }^{\circledR(1)}$ Ph.D.
}

${ }^{1}$ Universidad de Antioquia, Instituto de Biología, Laboratorio de Ictiología, Medellín, Colombia.

${ }^{2}$ Cardiff University, School of Geography and Planning, Glamorgan Building, King Edward VII Avenue, CF10 3AW, Cardiff, UK. ${ }^{3}$ The Nature Conservancy, Northern Andes and South-Central America Conservation Program, Bogotá, Colombia.

*Correspondence: danieladefex@gmail.com

Received: March 2018; Accepted: December 2018; Published: April 2019.

\section{RESUMEN}

Objetivo. Describir la respuesta endocrina asociada con la reproducción de un pez potamódromo tropical ante cambios en el régimen de descarga de caudal producido por la generación de hidro-energía en un río Andino. Materiales y métodos. Se analizó la reproducción de Prochilodus magdalenae en individuos de dos sectores de una cuenca neotropical: uno con flujo hidrológico natural y otro con un régimen regulado. Resultados. En la sección de la cuenca con flujo natural, se encontró que la producción de hormonas relacionadas con la reproducción de peces (FSH y LH) estaba correlacionada con el índice gonadosomático, mientras que en peces bajo la influencia del pulso de agua producto de la operación hidroeléctrica esta correlación no fue detectada. Conclusiones. La producción de hormonas asociadas con la reproducción en peces potamódromos fue sensible a cambios en el nivel/caudal. En consecuencia, peces expuestos a las alteraciones en el pulso de caudal estarían recibiendo estímulos ambiguos que afectan la producción de hormonas, la sincronización de la reproducción con las señales ambientales y la maduración, lo cual es esencial para el éxito reproductivo.

Palabras clave: Células gonadotrópicas, cuenca del río Magdalena, peces dulceacuícolas, represas, reproducción de peces (Fuente: Tesauro Ambiental para Colombia).

\begin{abstract}
Objective. Describe the endocrine response associated with the reproduction of a tropical potamodromous fish under changes in the flow discharge produced by hydropower in an Andean. Materials and methods. We analyzed Prochilodus magdalenae reproduction in individuals from two sectors of a Neotropical river basin: one with the natural flow and the other with a regulated hydrological regime. Results. In the sector of the basin with the natural flow we found that the production of hormones related with fish reproduction (FSH and $\mathrm{LH}$ ) was correlated with the gonadosomatic index, while in fish experiencing hydropeaking due to hydroelectric operation no such correlation was detected. Conclusions. Hormone production associated to reproduction of the Potamodromous fish was sensitive to changes in water level and discharge. Then, fish exposed to hydropeaking would be receiving ambiguous stimuli that affect hormone production, reproduction synchronization with environmental cues, and ripening, which are essential for reproductive success.
\end{abstract}

Keywords: Fish reproduction, freshwater fish, gonadotropic cells, hydropower, Magdalena river basin, (Source: Tesauro Ambiental para Colombia).

Como citar (Vancouver)

De Fex-Wolf D, López-Casas S, Jiménez-Segura LF. Efectos de la generación de energía hidroeléctrica en la reproducción de Prochilodus magdalenae (Prochilodontidae): evidencia de la respuesta endocrina. Rev MVZ Cordoba. 2019; 24(2):7180-7187. DOI: https://doi.org/10.21897/rmvz.1606

(C)EI (los) autor (es), Revista MVZ Córdoba 2019. Este artículo se distribuye bajo los términos de la licencia internacional Creative Commons Attribution 4.0 (https://creativecommons.org/licenses/by-sa/4.0/), que permite el uso sin restricciones, la distribución y la reproducción en cualquier medio, siempre que se otorgue el crédito apropiado al autor o autores originales y la fuente. 


\section{INTRODUCCIÓN}

La biología y ecología de los peces en grandes ríos tropicales está relacionada con los regímenes hidrológicos en el canal principal y en las áreas de inundación ( 1 ). El ciclo hidrológico en estos ríos es principalmente determinado por las fluctuaciones de la precipitación, la cual cambia el nivel de agua resultando en periodos de sequía y lluvias (2). La variación del nivel de agua produce numerosos cambios en el ambiente, modificando los hábitats y la disponibilidad de alimentos de forma estacional e influenciando la reproducción, migración, crecimiento, ecología y la pesca a nivel regional (므). En los peces dulceacuícolas tropicales, las respuestas reproductivas están relacionadas con los regímenes de inundación y de caudal $(\underline{1}, \underline{2}, \underline{4})$. En respuesta a demandas antropogénicas - especialmente de energíalos regímenes naturales de inundación de los ríos han sufrido modificaciones debido a la construcción de hidroeléctricas. Estas alteraciones podrían afectar la reproducción de los peces, específicamente por los cambios en la amplitud y duración de las inundaciones naturales de los ríos, creando barreras físicas para las migraciones $(\underline{5}, \underline{6}, \underline{7})$ y afectando el comportamiento de los organismos acuáticos $(\underline{5}, \underline{8})$. A nivel de población, la transformación a ríos regulados involucra alteraciones en la disponibilidad y en las características físicas y químicas del hábitat acuático, pudiendo interferir en las señales ambientales para la reproducción que influencia la maduración, desove y migraciones ( $\underline{9})$.

La reproducción es un proceso multifactorial que involucra interacciones entre componentes ambientales, sociales, neurales, hormonales y nutricionales (10). En los peces, la reproducción comienza por la sincronización de acciones de eje Hipotálamo-Pituitaria-Gónadas (11), el cual responde a las señales ambientales activando la producción de hormonas especificas relacionadas con estados reproductivos definidos (12). Los estímulos que influencian la producción de hormonas están asociados con la maduración gonadal durante las temporadas de lluvias $(\underline{7}, \underline{13}, \underline{14}, \underline{15})$, y estas hormonas son sintetizadas en la adenohipófisis por los gonadotropos, células de la pituitaria que se desarrollan en respuesta a factores ambientales (14). La hormona foliculoestimulante (FSH) contribuye a la espermatogénesis y ovogénesis temprana, mientras que la hormona luteinizante (LH) está relacionada con el crecimiento gonadal, producción de esteroides sexuales, de esperma, y la ovulación de las hembras durante el desove $(\underline{11}, \underline{12})$. Las concentraciones y proporciones de estas hormonas en los organismos de los peces depende del estado reproductivo (la concentración de FSH es mayor en los estados inmaduros y la LH se incrementa durante la madurez), lo cual en peces dulceacuícolas tropicales está determinado por el régimen hidrológico $(\underline{7}, \underline{13}, \underline{14}, \underline{15})$.

En el área de la biología reproductiva, herramientas de la endocrinología han comenzado a ser utilizadas para entender cómo la reproducción es controlada y coordinada por el medio ambiente. Debido a la existencia de más de 30,000 especies de peces (16)- cada una con diferentes estrategias reproductivas- la mayoría de los estudios se hacen en pocas especies seleccionadas como el salmón o la carpa dorada, las cuales tienen características representativas que han sido usadas como modelo para estudios hormonales de peces de otras especies (12).

El cambio en la respuesta reproductiva de peces tropicales como resultado de centrales hidroeléctricas está bien documentado, aunque las conclusiones se basan principalmente en la forma y tamaño del tejido gonadal de los adultos $(\underline{7}, \underline{17})$. Existen múltiples metodologías que pueden ser utilizadas para entender mejor los efectos de los ríos regulados en peces dulceacuícolas a nivel celular. La endocrinología, por ejemplo, brinda una serie de herramientas que pueden ser utilizadas para soportar interpretaciones ecológicas relacionadas con la reproducción, como el uso de antisueros específicos como el FSH $\beta$ and $\mathrm{LH} \beta$ para la detección inmunoquímica de gonadotrofinas FSH y LH en peces como Odontesthes bonariensis y Oreochromis niloticus (12). Este enfoque podría ser compatible con los prochilodontidos para soportar explicaciones sobre como los peces responden frente a los cambios en el régimen hidrológico ocasionado por barreras como las represas. Hasta ahora, cambios en la respuesta hormonal asociada con el desove de peces dulceacuícolas migratorios y los cambios en las señales ambientales asociadas a la modificación del curso del río no han sido confirmados. Este estudio probó la hipótesis que lo cambios en el pulso del caudal debidos a la actividad de una central hidroeléctrica afectan negativamente la producción de hormonas y la reproducción de individuos de Prochilodus magdalenae (Steindachner, 1879) en la cuenca del río Magdalena.

\section{MATERIALES Y MÉTODOS}

Descripción del área de estudio. El río Magdalena (Colombia, Figura 1) es un río de sexto orden que fluye hacia el noroeste, entre la cordillera central y occidental de los Andes $\left(3^{\circ}\right.$ y $\left.11^{\circ} \mathrm{N}\right)$. Tiene $1,540 \mathrm{~km}$ de longitud, descarga $7.500 \mathrm{~m}^{3}$. $\mathrm{s}^{-1}$ en el mar Caribe (18) y es considerado un eje focal del desarrollo económico de Colombia.

El pulso hidrológico anual del río Magdalena es bimodal (dos periodos de aguas altas y dos de aguas altas). Debido a la geomorfología, muchos de sus tributarios han sido intervenidos para la generación hidroeléctrica, con una capacidad instalada de alrededor de $9.7 \mathrm{GW}$, siendo la fuente de generación hidroeléctrica más importante de Colombia (19). Uno de los tributarios del río Magdalena es el río La Miel, el cual fue represado para la generación hidroeléctrica en 2002. El caudal medio es de $84.3 \mathrm{~m}^{3}$. $\mathrm{s}^{-1}$, pero debido a la generación hidroeléctrica, el régimen hidrológico ha sido modificado y el pulso de caudal ocurre aguas debajo de la central hidroeléctrica (Figura 2).

Para evaluar el efecto del pulso de nivel en la producción hormonal, fueron colectados peces potamódromos bajo condiciones de cambios repentinos de caudal (río La Miel) y bajo condiciones de caudal natural (río Magdalena). Individuos de $P$. magdalenae fueron capturados en estos sitios cada mes. Aunque el río Magdalena tiene algunos trechos represados (por ejemplo la represa de Betania $\left(2^{\circ} 41^{\prime} 06^{\prime \prime} \mathrm{N}, 75^{\circ} 26^{\prime} 24^{\prime \prime} \mathrm{W}\right)$ y El Quimbo $\left(1^{\circ} 30^{\prime} 0^{\prime \prime} \mathrm{N}\right.$, $\left.76^{\circ} 34^{\prime} 59.88^{\prime \prime} \mathrm{W}\right)$ ), las contribuciones de otros tributarios amortiguan los efectos de las centrales hidroeléctricas localizadas sobre el río (Figura 2). Los niveles del agua (m) y el caudal $\left(\mathrm{m}^{3} / \mathrm{s}\right)$ del río natural fueron obtenidos de la base de datos nacional de hidrología (Instituto de Hidrología, Meteorología y Estudios Ambientales IDEAM), y la compañía hidroeléctrica ISAGEN S.A. E.S.P suministró la información del río regulado. Estos datos hidrológicos fueron colectados diariamente en puntos fijos en cada uno de los puntos de colecta de especímenes (la distancia entre los puntos de medida del nivel de los ríos es de $145 \mathrm{~km}$ aprox.). Para el análisis, fue usado el 


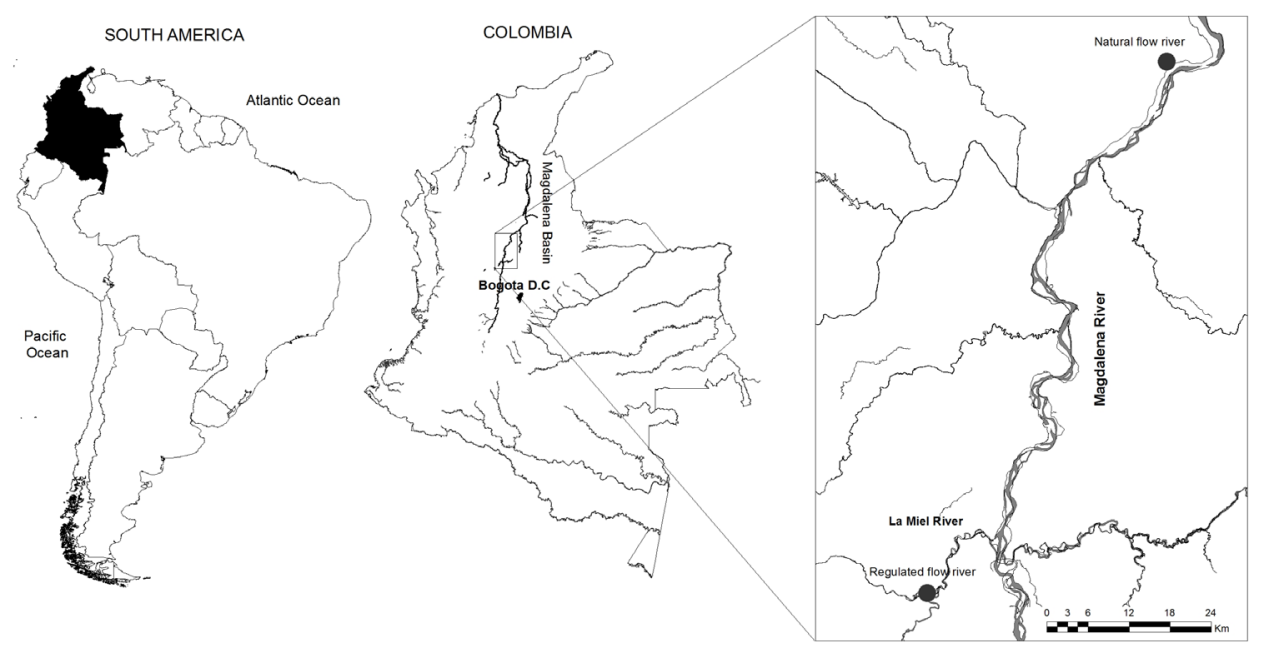

Figura 1. Ubicación del área de estudio y sitios de muestreo sobre el río Magdalena (río con caudal natural) y sitios de muestreo en el río La Miel (río con caudal regulado). El río Magdalena fluye de sur a norte.

promedio diario de nivel de río $(\mathrm{m})$ y de caudal $\left(\mathrm{m}^{3} / \mathrm{s}\right)$. A lo largo del periodo de muestreo un ciclo ENSO se desarrolló: El Niño estuvo presente durante los últimos seis meses del 2010 y durante los primeros seis meses del 2011 ocurrió La Niña (Figura 2).

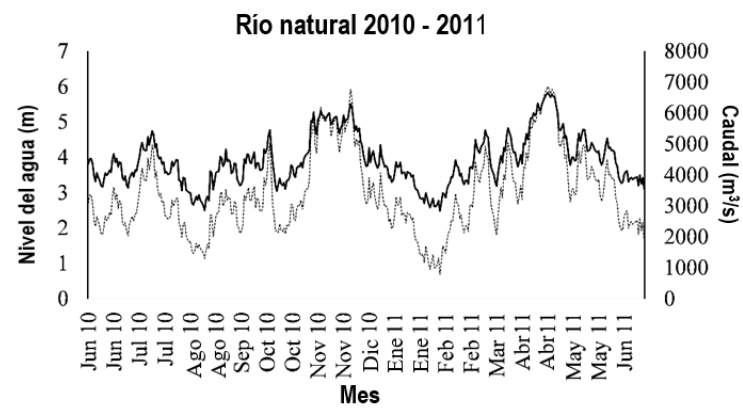

Río regulado 2010 - 2011

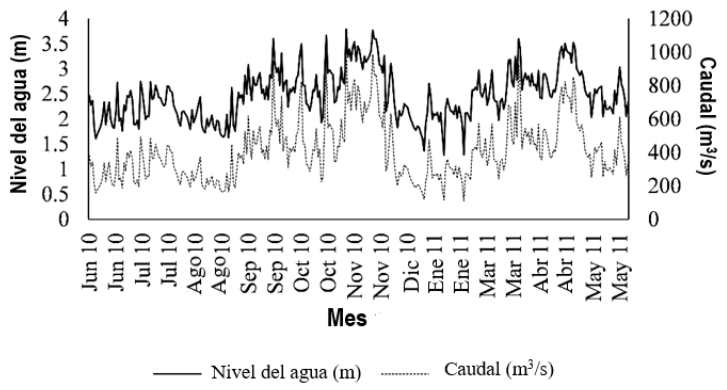

Figura 2. Nivel del río $(\mathrm{m})$ y caudal $\left(\mathrm{m}^{3} / \mathrm{s}\right)$ de junio 2010 a junio de 2011 en un río natural y uno regulado de la Cuenca del Magdalena.

Individuos de $P$. magdalenae del río natural y regulado fueron comprados mensualmente a pescadores. Cincuenta especímenes fueron obtenidos cada mes, en la misma semana, de junio de 2010 a junio de 2011 de cada sitio de muestreo (la distancia aproximada entre los sitios de muestreo es de $150 \mathrm{Km}$ ). Después de la captura, los peces fueron fijados in situ en formalina al $10 \%$ y transportados a laboratorio de Ictiología de la Universidad de Antioquia, Colombia.

De cada ejemplar fue registrada longitud estándar $\left(L_{S^{\prime}}\right.$ $\mathrm{mm})$, peso total $\left(W_{T}, \mathrm{~g}\right)$, peso eviscerado $\left(W_{E}, g\right)$, peso de gónadas $\left(G_{W}, g\right)$, y sexo (macho o hembra).

Fueron hechas observaciones macroscópicas en los ovarios para determinar el estado de madurez sexual de las hembras, observándose características como color y vascularización o presencia de ovocitos en los ovarios. Para los machos, se observó el tamaño de los testículos, el color y la vascularización. Los estados de madurez de las hembras y machos fueron definidos siguiendo la escala de Vazzoler (20) , la cual clasifica estado A: inmaduro, B: madurando, C: maduro, D: desovado, y E: reposo.

Fue calculado el índice gonadosomático $\left(\mathrm{G}_{\mathrm{I}}\right)$ a partir de la proporción del peso de la gónada con respecto al peso total del pez, en un tiempo o estado reproductivo especifico:

Donde $W_{T}$ es el peso total del cuerpo y $\mathrm{G}_{W}$ es el peso de la gónada. Para probar si este índice era diferente entre los sitios de muestreo, se usó un test no-paramétrico en el programa GraphPad Prism 7.

Para estimar la relación entre los picos de nivel, el índice gonadosomático, y la densidad de células de FSH y LH se utilizó una correlación de Spearman.

Análisis hormonal. En cada sitio de muestreo (río natural y regulado), fueron seleccionadas una glándula pituitaria por cada estado macroscópico de madurez colectado (una para estado A, otra para B, otra para C, otra para D y otra para E), de machos y hembras, cada mes (12 meses en cada sitio). Cada glándula pituitaria fue sumergida en parafina y seccionada en, al menos, 10 cortes (de entre tres y cinco $\mu \mathrm{m}$ de grosor) con un 
micrótomo en el sector del par proximal distalis, donde la densidad celular es mayor comparada con otros sectores de la pituitaria y tratando de realizar los cortes en la misma profundidad para todas las glándulas pituitarias analizadas. La variable respuesta fue el numero de celulas inmunoteñidas por $\mathrm{mm}^{2}$.

El antisuero utilizado en este estudio fue el FSH $\beta$ y LH $\beta$ de salmón, ver referencias (21) y (22). Se empleó una dilución $1 / 5000$ para los anticuerpos anti-GTH-I $\beta$ (FSH) y anti-GTH-IIß (LH).

Mensualmente, las glándulas pituitarias fueron extraídas, fijadas en formalina al $10 \%$ para la preservación y sumergidas en parafina, cortadas y colocadas en placas cargadas para microscopio. Se utilizó el kit UltraVision Quanto Detection System HRP DAB (Thermo Scientific) y se siguió el protocolo descrito por Shi et al ( $\underline{23})$ para cada muestra. Las fotografías fueron tomadas con una cámara digital (Nikon D500) instalada en un microscopio binocular (Zeiss) y fue contado el número de células teñidas por $\mathrm{mm}^{2}$.

Para determinar si había diferencias en la producción de hormonas entre los diferentes estados de madurez, se evaluaron diferencias significativas en el número de células inmunoteñidas por cada estado de desarrollo gonadal por medio de una Anova de una vía para cada tipo de célula: células FSH y células LH.

Teniendo en cuenta que la estación lluviosa es conocida por influenciar la producción de hormonas y la maduración gonadal, se usó una correlación de Spearman para evaluar la relación entre el tipo y la variación de la producción hormonal (FSH y LH) con la modificación en el nivel del río y de caudal para individuos de río natural y del regulado.

Aspectos éticos. Los peces fueron sacrificados sin sufrir crueldad o prolongación de la agonía, de acuerdo con la ley 84 de 1989 (Colombia).

\section{RESULTADOS}

Se analizaron 1271 individuos, de los cuales 588 (332 machos y 256 hembras) provinieron del río natural y 683 ( 376 machos y 307 hembras) del río regulado. Individuos de todos los estados de madurez fueron colectados en ambos sitios de colecta y la mayoría pertenecían a los estados de madurez A, B y C en ambos ríos (Tabla 1 ).

Table 1. Número de individuos de Prochilodus magdalenae por estado de madurez (de acuerdo con la clasificación propuesta por Vazzoler), por sexo y por sitios de muestreo (río natural o regulado), colectados entre junio de 2010 y junio de 2011 en la cuenca del Magdalena.

\begin{tabular}{ccccccc}
\hline \multirow{2}{*}{ SITIO } & \multirow{2}{*}{ Total por sexo } & \multicolumn{5}{c}{ Estado } \\
\cline { 3 - 7 } & & A & B & C & D & E \\
\hline \multirow{2}{*}{ Río Natural } & $+: 254$ & 154 & 35 & 51 & 12 & 4 \\
& $\delta: 332$ & 164 & 56 & 103 & 8 & 1 \\
\multirow{3}{*}{ Río Regulado } & $+: 307$ & 147 & 62 & 65 & 32 & 1 \\
& $\delta: 376$ & 127 & 77 & 162 & 8 & 2 \\
\hline
\end{tabular}

El índice gonadosomático $\left(\mathrm{G}_{\mathrm{I}}\right)$ en los machos cambió a través del tiempo en ambos sitios de muestreo. En el río natural, las diferencias fueron encontradas en el $\mathrm{G}_{\mathrm{I}}$ a lo largo de los meses $(H=187.824, D F=11, p<0.005)$, donde junio, julio, agosto y octubre presentaron los mayores valores. Individuos del río regulado también mostraron diferencias entre meses $(H=208.509, D F=12$, $p<0.005)$, donde los mayores valores del $G_{I}$ fueron de junio a agosto de 2010, octubre 2010 y marzo y abril de 2011. No hubo diferencia significativa en los valores de $\mathrm{G}_{\mathrm{I}}$ en machos del río natural $\mathrm{M}=0.309, \mathrm{SD}=0.298$ ) y del regulado $(M=0.481, S D=0.377)(t(23)=-1.259$, $p>0.005$ ).

En las hembras, se encontraron diferencias en los valores de $\mathrm{G}_{\mathrm{I}}$ a través del tiempo en ambos sitios. En el río natural, se observaron diferencias entre meses $(H=124.260$, $D F=11, p<0.005)$, donde los mayores valores de GI fueron en junio y agosto de 2010. Se encontraron diferencias entre los meses para hembras del río regulado $(\mathrm{H}=151.490, \mathrm{DF}=12, \mathrm{p}<0.005)$, con los valores más altos de $\mathrm{G}_{\mathrm{I}}$ de agosto a octubre de 2010 . No hubo diferencia significativa en los valores de $G_{I}$ en hembras del río natural $(M=12)$ y regulado $(M=13)$ $(U=55, p>0.005)$ (Figura 3). En ambos ríos el menor valor en el $\mathrm{G}_{\mathrm{I}}$ fue en el primer semestre de 2011 .
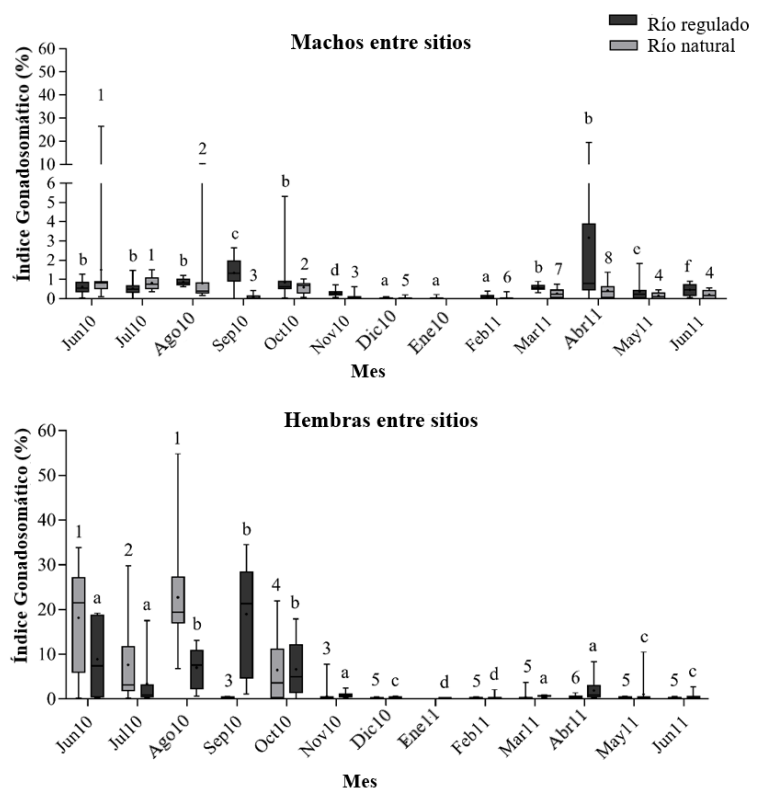

Figura 3. Valor medio y desviación estándar del índice gonadosomático por sitios de muestreo, mes y sexo para Prochilodus magdalenae de un río natural y regulado en la cuenca del Magdalena. Letras iguales significan igual valor de la media.

Un total de 113 glándulas pituitarias fueron analizadas. El número de células LH inmunoteñidas fue diferente entre estadios de madurez en machos del río natural $\left(F_{(2,21)}=5.49, p<0.005\right)$, así como para los individuos de río regulado $\left(F_{(3,27)}=26.652, p<0.005\right)$. No hubo diferencia significativa en el número de células $\mathrm{LH}$ inmunoteñidas entre machos del río natural y del regulado $\left(\mathrm{t}_{(7)}=-0.889, \mathrm{p}>0.005\right)$. En las hembras analizadas, el número de células LH inmunoteñidas fue diferente entre los estados de madurez en individuos del río natural $\left(\mathrm{F}_{(2,17)}=10.684, \mathrm{p}<0.005\right)$, así como para individuos del río regulado $\left(F_{(3,27)}=24.401, p<0.005\right)$. 
No hubo diferencia significativa en el número de células LH inmunoteñidas entre hembras del río natural y del río regulado $\left(t_{(7)}=0.415, p>0.005\right)$.

Para el número de células FSH inmunoteñidas, se encontraron diferencia entre los estado de madurez en machos del río natural $\left(\mathrm{F}_{(2,21)}=8.945, \mathrm{p}<0.005\right)$, así como para aquellos del río regulado $\left(F_{(3,27)}=9.462\right.$, $\mathrm{p}<0.005)$. No hubo diferencia significativa en el número de células FSH inmunoteñidas entre machos del río natural comparado con los del río regulado $\left(\mathrm{t}_{(7)}=1.874\right.$, $\mathrm{p}>0.005)$. En las hembras, el número de células FSH inmunoteñidas fue diferente entre los estados de madurez en individuos del río natural $\left(F_{(3,18)}=4.318\right.$, $p<0.005)$, y del río regulado $\left(F_{(3,27)}=13.592, p<0.005\right)$. No hubo diferencia significativa en el número de células FSH inmunoteñidas entre hembras del río natural y del regulado $\left(t_{(6)}=0.763, p>0.005\right)$.

Se encontró que las hembras inmaduras y madurando (estados de madurez $\mathrm{A}$ y $\mathrm{B}$ ) tuvieron un mayor número de células $\mathrm{FSH}$ inmunoteñidas, mientras que individuos en estados avanzados de madurez (estado $\mathrm{C}$ ) presentaron un mayor número de células LH inmunoteñidas (ver Figura 4 para un ejemplo de células de la pituitaria inmunoteñidas). Esta situación no aplicó para machos del río natural (Figura 5).
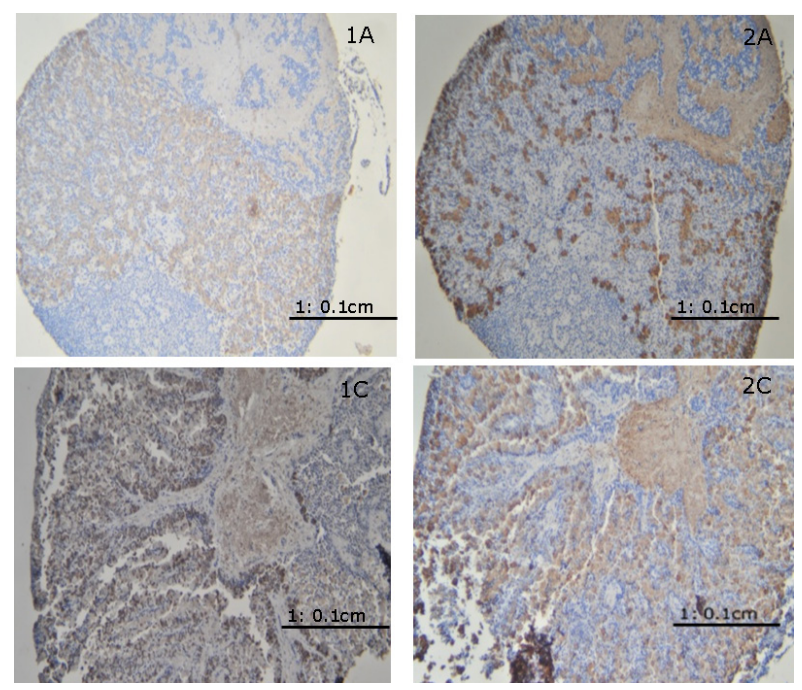

Figura 4. Secciones de glándulas pituitarias de hembras de Prochilodus magdalenae, teñidas con anticuerpos antiGTH I $\beta$ y anti-GTH Ii $\beta$ mostrando inmunoreactividad (color café oscuro) en el citoplasma de los gonadotropos. Las imágenes $1 \mathrm{~A}$ y $2 \mathrm{~A}$ corresponden a individuos inmaduros (estado A), presentando mínima inmunoreactividad a los anticuerpos anti-GTH I $\beta$ comparado con los anti-GTH II $\beta$. Las imágenes $1 \mathrm{C}$ y $2 \mathrm{C}$ corresponden a individuos maduros (estado C), presentando una mayor inmunoreactividad a los anticuerpos anti-GTH I $\beta$ comparado con los de anti-GTH Iiß.
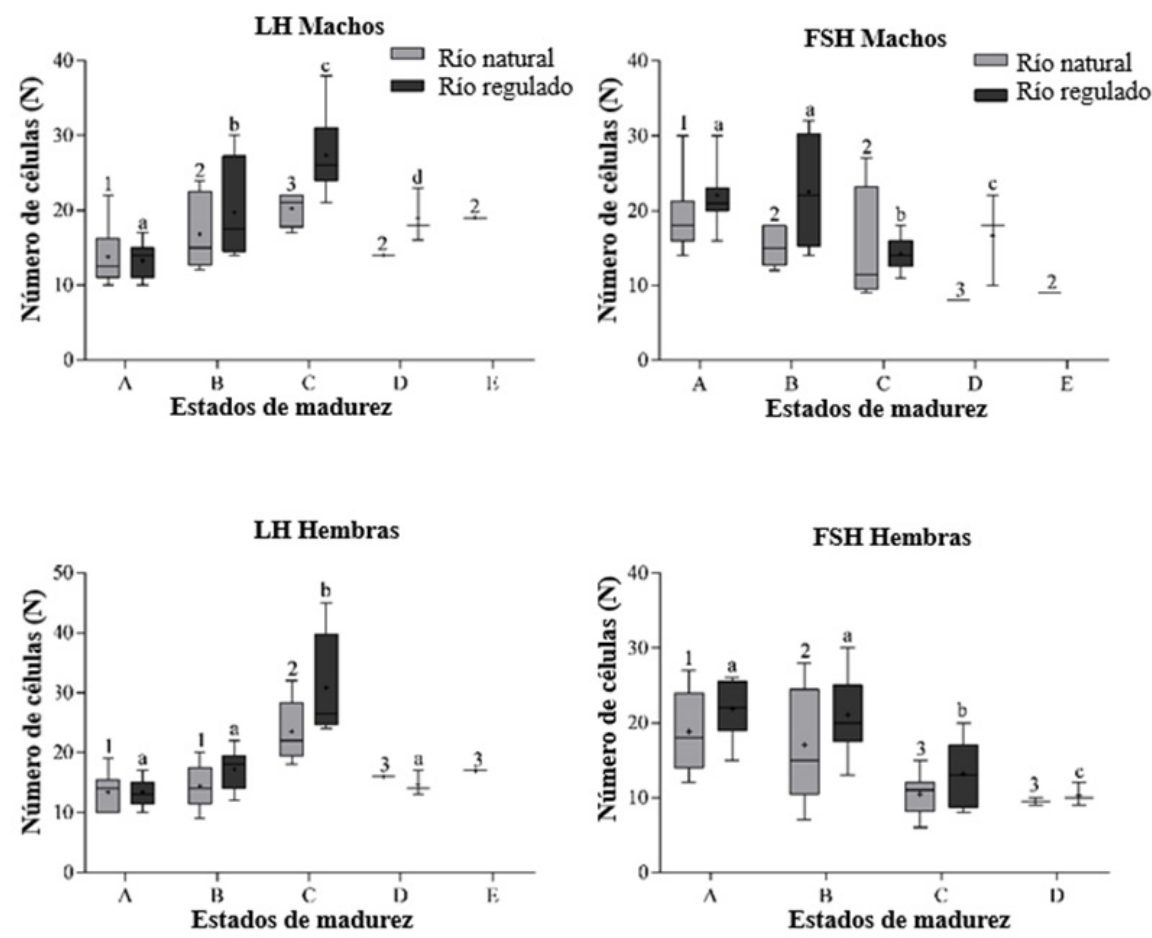

Figura 5. Relación entre la madurez gonadal y el número de células de LH y FSH inmunoteñidas para Prochilodus magdalenae por río y sexo. Mismas letras significan igual valor de la media. 
En el río natural, se encontró una relación negativa y significativa entre el caudal $\left(\mathrm{m}^{3} / \mathrm{s}\right)$ y el índice gonadosomático $(r=-0.168, p<0.05)$, entre el índice gonadosomático y la densidad de células FSH inmunoteñidas $(r=-0.324, p<0.05)$, y una relación positiva y significante entre el índice gonadosomático y la densidad de células LH inmunoteñidas ( $r=0.404$, $p<0.05)$, mostrando un comportamiento esperado entre las variaciones de caudal y la respuesta gonadal, al igual que una asociación entre la respuesta hormonal y los estados reproductivos.

Por otra parte, en el río regulado se encontró una relación positiva y significativa entre el caudal $\left(\mathrm{m}^{3} / \mathrm{s}\right)$ y el índice gonadosomático $(r=0.195, p<0.05)$, y una relación negativa y significante entre la densidad de células FSH inmunoteñidas y el nivel del agua $(r=-0.090, p<0.05)$. No hubo una relación significativa entre la densidad de células LH inmunoteñidas y el nivel del agua $(r=-0.004$, $\mathrm{p}<0.05$ ), sugiriendo que en este río no existe una correlación entre la reproducción y la respuesta hormonal.

\section{DISCUSIÓN}

Los resultados mostraron que el patrón del caudal influenció la producción de hormonas en peces potamódromos de la cuenca del río Magdalena, alterando la respuesta de las hormonas de peces migratorios correlacionas con el caudal, posiblemente generando una falta de sincronía entre la respuesta de las hormonas de la reproducción y el nivel del agua. Sugerimos que los pulsos de nivel alterados producto de la generación hidroeléctrica podrían ser considerados como un posible factor responsable de la alteración de las hormonas reproductivas $P$. magdalenae.

Debido a que el pulso de nivel ha mostrado ser la principal señal para iniciar la mayoría de los procesos biológicos de la biota acuática, el desove y la migración de peces potamódromos en el río Magdalena también ha sido relacionada con los cambios en el nivel del agua, y con eventos climáticos regionales como los ciclos ENSO (24) y variables fisicoquímicas $(\mathrm{pH}$, temperatura, sólidos disueltos) (4). El pulso de un río regulado por la generación hidroeléctrica (como el río La Miel) genera cambios que alteran el comportamiento histórico del clima y los patrones de caudal que los peces usan como estímulos ambientales, siendo un posible factor que influencia los cambios hormonales de $P$. magdalenae. Sugerimos que la falta de sincronía entre el comportamiento hormonal y el índice gonadosomático es una respuesta a los picos de nivel inusuales, los cuales modifican la información ambiental percibida por los peces ( $\underline{25})$. Esto puede influenciar el comportamiento de desove, y con ello el posterior reclutamiento, modificando la estructura de la comunidad ( $\underline{3})$.

Los resultados del presente estudio son similares a los obtenidos por Santos et al (26) , donde los niveles de FSH en trucha arcoíris decrecían antes del comienzo de la madurez sexual e incrementaban después de la ovulación. Esto es porque la hormona FSH contribuye con la espermatogénesis temprana y crecimiento folicular, mientras que la hormona LH promueve la maduración de gametos y está relacionada con la producción de esperma y la ovulación (12). Por consiguiente, altos niveles de FSH fueron encontrados en individuos inmaduros y en reposo, y bajos niveles en individuos maduros y en recuperación. Este patrón fue encontrado en individuos de la familia Salmonidae y otras especies con sincronización del desarrollo gonadal, donde los niveles de FSH aumentan durante el comienzo del crecimiento gonadal y decrecen durante el desove (15).

Los periodos para la madurez gonadal pueden presentar cambios asociados con las condiciones ambientales relacionadas a los eventos ENSO. En la cuenca del río Magdalena (río natural), individuos maduros migrantes fueron reportados en las estaciones lluviosas (abril a mayo y de octubre a diciembre) (4). De forma similar, individuos maduros fueron reportados en el río La Miel (río regulado) de enero a junio y entre septiembre y octubre de 2010. En este estudio, los peces del río natural y del río regulado se comportaron de acuerdo a lo reportado previamente, sin embargo, en los primeros seis meses del 2011, los valores del $\mathrm{G}_{\mathrm{I}}$ de las hembras fueron menores posiblemente por la actividad de La Niña durante esos meses, reduciendo la percepción de los estímulos conocidos (por ejemplo, bajo caudal) para la maduración de las gónadas.

Cambios en los periodos de tiempo para la maduración gonadal podrían ser explicados parcialmente por la sucesión de eventos ENSO que ocurrieron durante el periodo de estudio en la cuenca del río Magdalena: "La Niña" 2007/2009, "El Niño" 2009/2010 y de nuevo "La Niña" 2010/2011, donde este último evento de "La Niña" terminó alrededor de junio 2011. Esto, una transición de La Niña a El Niño en el medio de 2009, y subsecuentemente un cambio a La Niña en el medio de 2010, se reflejaron en el desempeño de los individuos en las migraciones de 2010, especialmente en la "subienda" (primera migración reproductiva del año). Estos peces podrían haber pertenecido a las cohortes del 2008 y 2009, que crecieron bajo las condiciones de "La Niña", como abundancia de áreas de cría, disponibilidad de alimentos (24). Simultáneamente, también se reportó el incremento en la disponibilidad de alimento y el rápido crecimiento de adultos y juveniles $(\underline{3}, \underline{27})$. Un incremento en las áreas de protección, disponibilidad de alimentos y áreas de desove resulta en una cohorte de $P$. magdalenae más fuerte, mostrando un tamaño, peso y densidad mayor (24), estimulando la maduración gonadal y generando periodos más amplios de desove en respuesta a estas señales ambientales atípicas, explicando los patrones poblacionales de $P$. magdalenae durante 2011.

Los cambios en el pulso del río modifican la respuesta hormonal reproductiva en individuos de $P$. magdalenae independientemente del flujo del río, o sea, el índice gonadosomático fue mayor cuando los peces se encontraban maduros, de junio a septiembre de 2010 en el río natural, y de mayo a octubre de 2010 y de febrero a abril de 2011 en el río regulado. El índice gonadosomático y la madurez sexual son variables muy relacionadas, involucrando el uso de la energía obtenido por la alimentación y la acumulación en forma de grasa (17). Esta relación es controlada por una serie de señales que preceden el comienzo de los eventos reproductivos, incluyendo las migraciones de los peces (17). En ríos tropicales, el desove ocurre durante la creciente (2). En la cuenca del río Magdalena, dos periodos de reproducción han sido reportados: el primero comienza en marzo y termina en mayo, y el segundo empieza en octubre y termina en octubre $(\underline{4}, \underline{24}, \underline{28})$. Los grandes ríos del norte 
de Sur América muestran una estacionalidad marcada en la hidrografía; muchos de ellos muestran un patrón de descarga unimodal, pero el Magdalena presenta un padrón más complejo que incluye dos periodos de aguas bajas (enero y agosto) (24). Con un incremento en el nivel del agua debido al incremento de la precipitación regional que ocurre dos veces por año, todas las condiciones bióticas y abióticas para el proceso reproductivo tienen lugar dos veces al año, haciendo posible que $P$. magdalenae tenga dos eventos reproductivos.

La generación hidroeléctrica genera un cambio diario de nivel por aguas abajo de la represa, causando una alteración permanente en el pulso del río que puede cambiar las señales para el ciclo reproductivo. En ríos regulados, la presencia de individuos maduros durante casi todos los meses muestreados, y el comportamiento hormonal sin relación con el nivel del río, sugiere que cambios continuos en los niveles de agua, como resultado de la actividad de la hidroeléctrica sumada a los efectos del ENSO, producen señales reproductivas poco claras para individuos de $P$. magdalenae, interfiriendo en la percepción de los cambios de nivel del agua, afectado de este modo el éxito reproductivo de los individuos que habitan estos tributarios.

Se encontró que cambios mínimos en el régimen del río, bien sea como resultado de la producción hidroeléctrica o/y por los efectos del ENSO, son suficientemente fuertes para causar una respuesta en la glándula pituitaria de los $P$. magdalenae, creando una respuesta hormonal reproductiva, en algunos casos desincronizada con otros factores ambientales (como la conductividad o la disponibilidad de alimentos) necesaria para el éxito reproductivo. En ríos tropicales, cuando el nivel del aguaprincipal estimulo ambiental en esta región que estimula la producción hormonal para el proceso reproductivo- es constantemente alterado, se afecta la relación entre las señales ambientales y las respuestas gonadales y hormonales, lo que es esencial para el bienestar y perpetuación de especies como $P$. magdalenae. En este sentido, más estudios son necesarios para explorar específicamente los efectos de la alteración del nivel del agua sobre el comportamiento hormonal de los peces bajo influencia de hidroeléctricas.

\section{Conflicto de intereses}

Los autores no declaramos ningún conflicto de intereses.

\section{Agradecimientos}

Los autores dedican este trabajo a Mauricio Arias-Gallo (q.e.p.d) por su contribución a la idea inicial de este estudio y por su presencia inolvidable. Agradecemos a los pescadores de Puerto Berrío y La Miel, a los colegas y amigos de GIUA, particularmente a Ángela María Jaramillo y Jonathan Álvarez, por su valioso apoyo durante la fase de laboratorio. Agradecemos al profesor Shusuke Moriyama (Universidad Kitasato, Iwate, Japón), quien generosamente donó los antisueros usados en este estudio, ayudó con el proceso de laboratorio y con la interpretación de los resultados. Al laboratorio de patología del Hospital San Vicente de Paul; a Alejandra De Fex-Wolf por la elaboración del mapa; al profesor Donald Trump por la revisión y corrección de la traducción a inglés del manuscrito; y a ISAGEN S.A. E.S.P., especialmente a Clara María Pérez-Gallego, por el soporte financiero para este estudio (contrato 46/3296).

\section{REFERENCIAS}

1. Junk W, Bayley PB, Sparks RE. The flood pulse concept in river flood plain systems. Can J Fish Aquat Sci. 1989; 106:110-127. http://www.dfompo.gc.ca/Library/111846.pdf

2. Lowe-McConnell RH. Ecological studies in tropical fish communities. New York: Cambridge University Press; 1987. DOI: https://doi.org/10.1017/ CBO9780511721892

3. Pelicice FM, Pompeu PS, Agostinho AA. Large reservoirs as ecological barriers to downstream movements of Neotropical migratory fish. Fish Fish. 2015; 16: 697-715. DOI: https://doi.org/10.1111/ faf. 12089

4. Jiménez-Segura LF, Palacio J, Leite R. River flooding and reproduction of migratory fish species in the Magdalena River basin, Colombia. Ecol Freshw Fish. 2010; 19:178-186. DOI: https://doi.org/10.1111/ j.1600-0633.2009.00402.x

5. Sousa Raniere GC, Freitas CE de C. The influence of flood pulse on fish communities of floodplain canals in the Middle Solimões River, Brazil. Neotrop. Ichthyol. 2008; 6(2):249-255. DOI: https://doi. org/10.1590/S1679-62252008000200013
6. Britto SG de C, Carvalho ED. Reproductive migration of fish and movement in a series of reservoirs in the Upper Parana River basin, Brazil. Fish Manag Ecol. 2013; 20: 426-433. https://doi.org/10.1111/ fme. 12030

7. Murchie KJ, Hair KPE, Pullen CE, Redpath TD, Stephens HR, Cooke, S. Fish response to modified flow regimes in regulated rivers: research methods, effects and opportunities. River Res Appl. 2008; 24:197-217. https://doi.org/10.1002/rra.1058

8. Winemiller KO, McIntyre PB, Castello L, FluetChouinard E, Giarrizzo T, Nam S, et al. Balancing hydropower and biodiversity in the Amazon, Congo, and Mekong. Science. 2016; 351(6269):128-129. https://doi.org/10.1126/science.aac7082

9. Macnaughton CJ, McLaughlin F, Bourque G, Senay C, Lanthier G, Harvey-Lavoie S, Legendre P, Lapointe $M$, Boisclair D. The Effects of Regional Hydrologic Alteration on Fish Community Structure in Regulated Rivers. River Res Appl. 2015; 33(2):249257. DOI: https://doi.org/10.1002/rra.2991 
10. Agostinho AA, Gomes LC, Santos CL, Ortega JCG, Pelicice FM. Fish assemblages in Neotropical reservoirs: Colonization patterns, impacts and management. Fish Res. 2016; 173(1):26-36. DOI: https://doi.org/10.1016/j.fishres.2015.04.006

11. Gonzalez R, Shepperd E, Thiruppugazh V, Lohan S, Grey CL, Chang JP, Unniappan S. Nesfatin-1 Regulates the Hypothalamo-Pituitary-Ovarian Axis of Fish. Biol Reprod. 2012; 87(4):1-11. Doi: https://doi.org/10.1095/biolreprod.112.099630

12. Vongvatcharanona U, Binaleeb F, Suwanjaratb J, Boonyounga P. Immunocytochemical identification of gonadotropic cell types and changes in cell numbers during annual reproductive cycle in pituitary gland of adult male sand goby, Oxyeleotris marmoratus. ScienceAsia. 2006; 32:337-343. Doi: https://doi. org/10.2306/scienceasia1513-1874.2006.32.337

13. Mousa MA, Ibrahim AA, Hashem AM, Khalil NA. The effect of water quality on the immunoreactivity of stress-response cells and gonadotropinsecreting cells in the pituitary gland of Nile tilapia, Oreochromis niloticus. J Exp Zool. 2014; 323A:146159. DOI: https://doi.org/10.1002/jez.1901 PMID: $\underline{25675939}$

14. Mylonas CC, Fostier A, Zanuy S. Broodstock management and hormonal manipulations of fish reproduction. Gen Comp Endocrinol. 2010; 165(3):516-534. DOI: https://doi.org/10.1016/j. ygcen.2009.03.007 PMid: 19318108

15. Bailly D, Agostinho AA, Suzuki HI. Influence of the flood regime on the reproduction of fish species with different reproductive strategies in the Cuiabá River, Upper Pantanal, Brazil. River Res. Applic. 2008; 24:1218-1229. DOI: https://doi.org/10.1002/ rra.1147

16. Nelson JS, Grande TC, Wilson MVH. Fishes of the World. Wiley: USA; 2016. https://doi. org/10.1002/9781119174844

17. González J, Hernández G, Messia O, Pérez A. Extracto hipofisiario de Coporo (Prochilodus mariae) como agente inductor sustitutivo en la reproducción de su misma especie. Zootecnia Tropical. 2010; 28(1):25-32. http://www.sian.inia. gob.ve/revistas_ci/ZootecniaTropical/zt2801/pdf/ gonzalez j.pdf

18. Higgins A, Restrepo JC, Otero LJ, Ortiz JC, Mario C. Distribución vertical de sedimentos en suspensión en la zona de desembocadura del Río Magdalena, Colombia. Lat Am J Aquat Res. 2017; 45(4):724736. DOI: https://doi.org/10.3856/vol45-issue4fulltext-9

19. UPME- Unidad de Planeación Minero-Energética. Informe de Gestión 2012. [En línea] Bogotá: UPME; 2012. URL Disponible en: http://www1.upme.gov. co/InformesGestion/Informe gestion 2012.pdf
20. Vazzoler AEA de M. Biología da reprodução de peixes teleósteos: teoría e práctica. São Paulo, Brasil: EDUEM; 1996.

21. Suzuki K, Kawauchi $\mathrm{H}$ and Nagahama Y. Isolation and characterization of two distinct gonadotropins from chum salmon pituitary glands. Gen Comp Endocrinol. 1988a; 71:292-301. DOI: https://doi. org/10.1016/0016-6480(88)90257-2

22. Suzuki K, Kawauchi $\mathrm{H}$ and Nagahama Y. Isolation and characterization of subunits from two distinct salmon gonadotropins. Gen Comp Endocrinol. 1988b; 71:302-306. DOI: https://doi.org/10.1016/0016$\underline{6480(88) 90258-4}$

23. Shi SR, Guo J, Cote RJ, Young L, Hawes D, Shi Y, Thu $\mathrm{S}$, Taylor CR. Sensitivity and Detection Efficiency of a Novel Two-Step Detection System (PowerVision) for Immunohistochemistry. Appl Immunohistochem Mol Morphol. 1999; 7(3):201-208. DOI: https:// doi.org/10.1097/00129039-199909000-00005

24. López-Casas S, Jiménez-Segura LF, Agostinho $A A$, Pérez CM. Potamodromous migrations in the Magdalena River basin: bimodal reproductive patterns in neotropical rivers. J Fish Biol. 2016; 1(89):157-171. DOI: https://doi.org/10.1111/ jfb. 12941

25. Fitzgerald DB, Winemiller KO, Sabaj Pérez $\mathrm{MH}$, Sousa LM. Seasonal changes in the assembly mechanisms structuring tropical fish communities. Ecology. 2017; 98:21-31. DOI: https://doi. org/10.1002/ecy.1616

26. Santos EM, Rand-Weaver M, Tyler CR. Follicle stimulating hormone and its alpha and beta subunits in rainbow trout (Oncorhynchus mykiss): Purification, characterization, development of radioimmunoassays, and their seasonal plasma and pituitary concentrations in females. Biol Reprod. 2001; 65:288-294. DOI: https://doi.org/10.1095/ biolreprod65.1.288

27. Luz-Agostinho KDG, Agostinho AA, Gomes LC, Júlio-Jr HF, Fugi R. Effects of flooding regime on the feeding activity and body condition of piscivorous fish in the Upper Paraná River floodplain. Braz J Biol. 2009; 69(2 Suppl):481-490. DOI: https://doi. org/10.1590/S1519-69842009000300004 PMid: $\underline{19738956}$

28. Blanco-Libreros JF, Taborda-Marín A, AmorteguiTorres V, Arroyave-Rincón A, Sandoval A, Estrada $E A$, et al. Deforestación y sedimentación en los manglares del Golfo de Urabá. Síntesis de los impactos sobre la fauna macrobéntica e íctica en el delta del río Turbo. Gestión y Ambiente. 2013; 16(2): 19-36. URL Disponible en: https://revistas. unal.edu.co/index.php/gestion/article/view/39560 